\title{
Dos décadas de estudio de la diversidad de hormigas en Colombia
}

\author{
Patricia Chacón de Ulloa*, Juan Carlos Abadía \\ Grupo de Investigación en Biología, Ecología y Manejo de Hormigas, Departamento de Biología, \\ Facultad de Ciencias Naturales y Exactas, Universidad del Valle, Cali, Colombia
}

\begin{abstract}
Resumen
El Grupo de Investigación en Biología, Ecología y Manejo de Hormigas de la Universidad del Valle ha publicado en las últimas dos décadas 60 estudios llevados a cabo en diferentes ecosistemas colombianos que abarcan paisajes rurales, áreas protegidas y zonas agrícolas y urbanas. El $70 \%$ de las contribuciones se concentró en la región Andina, seguida de las regiones Pacífica, Insular, Amazonia y Orinoquia, cada una con el 7 \%, y un $2 \%$ en la región Caribe. En cuanto al número de especies de hormigas registradas, sobresalieron las zonas con mayor esfuerzo de muestreo como las tierras bajas del Pacífico (227 especies), el valle interandino del río Cauca (215 especies), el piedemonte amazónico en el departamento de Caquetá (132 especies), el Parque Nacional Natural Gorgona (129 especies) y la región Caribe (122 especies). Los estudios incluyeron algunas especies de hormigas clave, ya sea por su rareza, endemismo, amplia distribución o comportamiento invasivo, y, así mismo, aquellas catalogadas como indicadoras de hábitats o usos del suelo, de diversidad y de perturbación del ecosistema. Igualmente, en los estudios se mencionan los servicios ecosistémicos a los que contribuyen varias especies.
\end{abstract}

Palabras clave: áreas protegidas, bioindicadores, riqueza de especies, paisajes rurales, servicios ecosistémicos.

Two decades of study of the diversity of ants in Colombia

\begin{abstract}
The Research Group on the Biology, Ecology, and Management of Ants at the Universidad del Valle has published 60 studies in the last two decades on different Colombian ecosystems, including rural zones, protected areas, crops, and urban areas. Seventy percent of the contributions concentrated in the Andean region, followed by the Pacific, Insular, Amazon and Orinoco regions, each with 7\%, and 2\% in the Caribbean region. In terms of number of species, the ant diversity recorded reflects areas with greater sampling effort, such as the Pacific lowlands (227 species), the Andean valley of the Cauca river (215 species), the Amazon piedmont in the department of Caquetá (132 species), the Gorgona Natural National Park (129 species), and the Caribbean region (122 species). Studies include some key species of ants due to their rarity, endemism, wide distribution, or invasive behaviour, as well as those classified as indicators of habitats and land uses, diversity, and ecosystem disturbance. Ecosystem services to which various species contribute are also mentioned.
\end{abstract}

Key words: Protected areas, bioindicators, species richness, rural landscapes, ecosystem services.

\section{Introducción}

Las hormigas están agrupadas en la familia Formicidae y el orden Hymenoptera, que incluye también a las abejas, avispas y avispas sierra (Bolton, et al., 2007). Se originaron hace alrededor de 120 millones de años (Brady, et al., 2006) y se han convertido en el grupo de insectos sociales más diverso y exitoso, con cerca de 12.500 especies descritas (Bolton, et al., 2007), divididas en 290 géneros y 21 subfamilias vivientes (Ward, 2007). Se distribuyen en todos los continentes a excepción de la Antártica y son organismos conspicuos de la mayoría de los ecosistemas terrestres, los cuales han alcanzado su mayor diversidad y biomasa en los trópicos (Fernández, 2003; Bolton, et al., 2007).
Las hormigas presentan un amplio rango de hábitos de forrajeo y de asociación con otros organismos (Hölldobler \& Wilson, 1990). Además, se las considera una de las herramientas más poderosas y útiles para vigilar cambios en los ecosistemas (Alonso, 2000) debido a su riqueza, abundancia, especialización, facilidad de muestreo, respuesta a cambios medioambientales, fidelidad ecológica, importancia funcional en un ecosistema y estrecha asociación con otras especies, así como al conocimiento taxonómico de la especie. Todos

\footnotetext{
*Correspondencia:

Patricia Chacón de Ulloa, patricia.chacon@correounivalle.edu.co

Recibido: 14 de febrero de 2014

Aceptado: 7 de abril de 2014
} 
estos elementos hacen de estos insectos el mejor grupo indicador (Majer, 1983; Brown, 1991), principalmente en estudios que involucran procesos de perturbación (Andersen \& Majer, 2004).

Desde comienzos de la década de los 90, el Grupo de Biología, Ecología y Manejo de Hormigas de la Universidad del Valle lleva a cabo estudios en diferentes líneas de investigación en este campo: biología y manejo, comunidades e indicadores, agroecología y ecología del paisaje (http://hormigas.univalle. edu.co/). El presente trabajo recopila las investigaciones del Grupo desarrolladas y publicadas bajo la orientación de la autora principal; se resaltan los principales aportes al conocimiento de la diversidad de las hormigas y su utilidad en los estudios ecológicos, así como al establecimiento de la línea de base para la toma de decisiones en el manejo y conservación de la biodiversidad.

\section{Recopilación de la información}

Se revisaron las publicaciones del Grupo de Biología, Ecología y Manejo de Hormigas en los últimos 21 años (1993-2014).Todas las contribuciones se agruparon según su ubicación en alguna de las seis regiones naturales de Colombia, incluida la región insular del Pacífico. Se examinaron las bases de datos y los resultados de cada trabajo con el fin de sintetizar la información sobre la riqueza y la diversidad de las especies de hormigas, el uso de algunas de ellas como indicadores biológicos, las especies comunes, las especies raras, las hormigas introducidas e invasoras y los servicios ecosistémicos que estos insectos prestan. Igualmente, se hizo una actualización taxonómica (http:// www.antweb.org) para lograr uniformidad y fiabilidad en los datos. Todas las colecciones de referencia se encuentran depositadas en el Museo de Entomología de la Universidad del Valle (MUSENUV).

La información sobre las localidades muestreadas en cada región (Figura 1) se detalla a continuación.

Región Caribe: trece municipios de los departamentos de Bolívar, Cesar, Córdoba y Magdalena, donde se evaluó la fauna de hormigas asociadas a cultivos de naranja con diferentes tipos de manejo agronómico. Las condiciones de altitud variaban entre los 20 y los 896 metros y la temperatura entre 28 y $37{ }^{\circ} \mathrm{C}$. (Abadía, et al., 2013).

Región Andina: en esta región se concentró el mayor esfuerzo de muestreo, con investigaciones en bosques montanos $\left(2.300 \mathrm{~m}\right.$ de altura y 12 a $\left.20{ }^{\circ} \mathrm{C}\right)$ como los de Hato Viejo en el Parque Nacional Natural Farallones de Cali (Bustos \& Ulloa-Chacón, 1996-97) y el corregimiento Borrero Ayerbe en el municipio de Dagua (Chacón de Ulloa, et al., 1996; Jones, et al., 1999), departamento del Valle del Cauca; los paisajes subandinos del municipio de Aranzazu en el departamento de Caldas (Abadía, et al., 2010), la
Reserva Forestal Natural de Bremen-La Popa en Quindío (Chaves, 2003; Chaves, et al., 2008) y el paisaje cafetero del departamento de Risaralda, en el municipio de La Celia (Zabala, et al., 2013).

La mayor área de estudio correspondió al bosque seco tropical del valle interandino del río Cauca, desde el centro de Risaralda hasta el norte del departamento del Cauca, con altitudes entre los 900 y los 1.100 metros y una temperatura promedio de $24{ }^{\circ} \mathrm{C}$. El objetivo principal de los estudios fue el efecto de la transformación del paisaje sobre la diversidad de hormigas en los fragmentos de bosque y sus matrices (Armbrecht \& Chacón de Ulloa, 1997; Armbrecht \& Chacón de Ulloa, 1999; Armbrecht, et al., 2001b; Chacón de Ulloa \& Armbrecht, 2006; Arcila-Cardona, et al., 2008; Chacón de Ulloa, et al., 2008), seguido por el análisis sobre las relaciones entre especies y área en nueve bosques (Lozano-Zambrano, et al., 2009), las relaciones biogeográficas de las hormigas ponerinas (Zabala, et al., 2008), los patrones de diversidad alfa y beta (Cabra-García, et al., 2012) y la síntesis de la riqueza taxonómica regional (Chacón de Ulloa, et al., 2012).

En los mismos fragmentos de bosque se han descrito, así mismo, las relaciones entre plantas y hormigas y entre estas e insectos heterópteros (Ramírez, et al., 2001), y la simbiosis entre hormigas y coleópteros estafilínidos (García, et al., 2001; García \& Chacón de Ulloa, 2005). Otros trabajos se enfocaron en la biología reproductiva, la ecología y el control de algunas especies nativas dominantes como la pequeña hormiga de fuego Wasmannia auropunctata (Armbrecht \& Ulloa-Chacón, 2003; Chacón de Ulloa, 2003a; Achury, et al., 2008; López, et al., 2008; Achury, et al., 2012), e invasoras, como la hormiga loca Nylanderia (Paratrechina) fulva (Chacón de Ulloa, et al., 1994; Aldana, et al., 1995a; Chacón de Ulloa, 1998; Chacón de Ulloa, et al., 2000; Arcila, et al., 2002a; Arcila, et al., 2002b) y la hormiga fantasma Tapinoma melanocephalum (Jaramillo \& Chacón de Ulloa, 2003). Los estudios sobre las hormigas como plagas potenciales en criaderos de mariposas (Sanabria-Blandón \& Chacón de Ulloa, 2009) y las hormigas en suelos cultivados con maracuyá (Arenas, et al., 2013), han contribuido al conocimiento del papel de algunas especies depredadoras en sistemas productivos. En cuanto a las hormigas urbanas, son trabajos pioneros en Colombia el inventario hecho en siete ciudades del Valle del Cauca (Chacón de Ulloa, et al., 1996; Chacón de Ulloa, 2003b; Chacón de Ulloa, et al., 2006; Achury, et al., 2011; Chacón de Ulloa \& Achury, 2011; Chacón de Ulloa, et al., 2013), el estudio sobre la importancia de algunas especies comunes como vectores de patógenos nosocomiales (Olaya, et al., 2005) y la evaluación de diferentes métodos de control químico de la hormiga fantasma (Ulloa-Chacón \& Jaramillo, 2004). Por último, cabe mencionar que el trabajo sobre la incidencia de la hormiga 


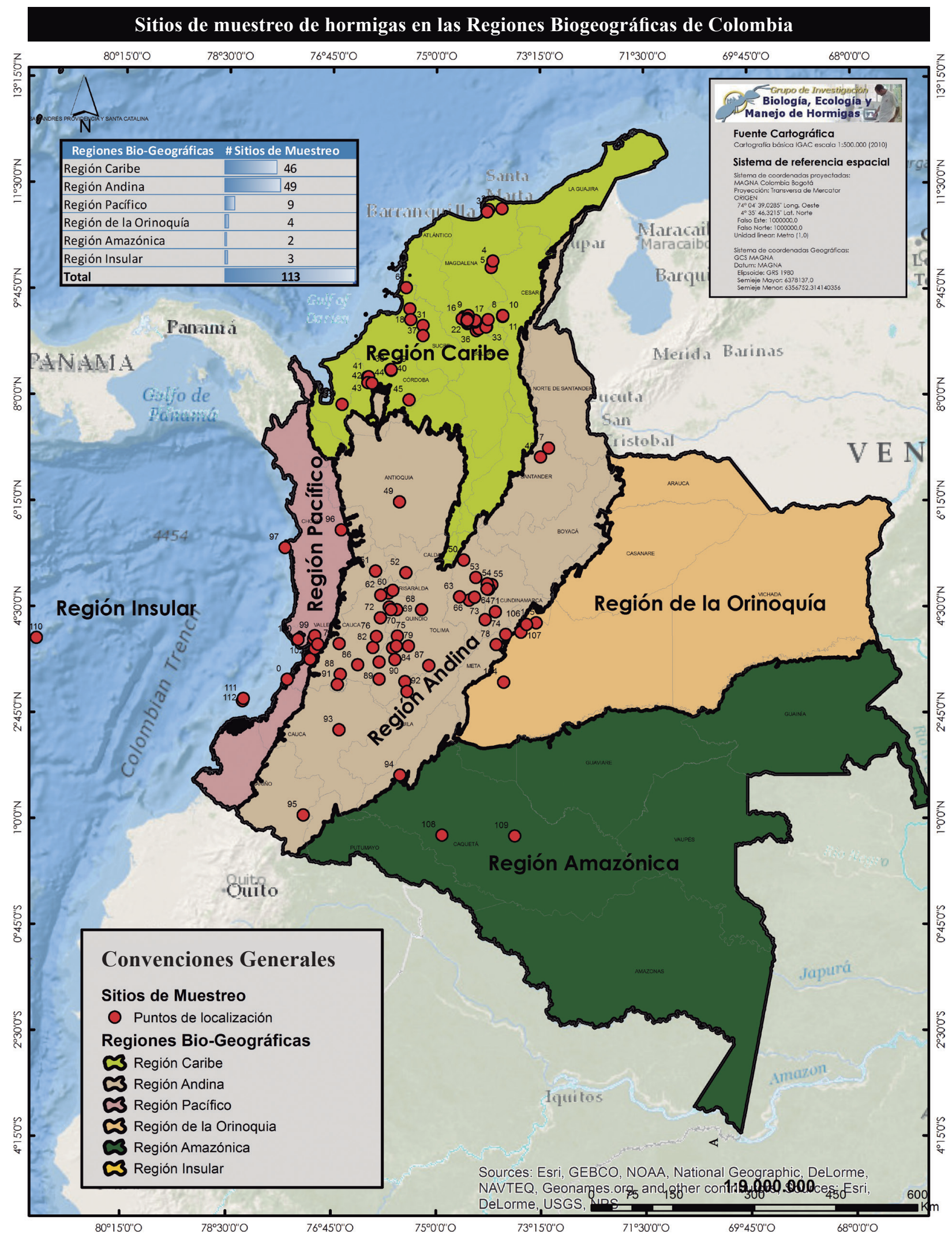

Figura 1. Sitios de muestreo de hormigas en las regiones naturales de Colombia. 
cortadora de hojas (Atta cephalotes) en zonas verdes de la ciudad de Cali (Montoya, et al., 2006) fue uno de los primeros estudios sobre esta especie en ambientes urbanos en Colombia (Chacón de Ulloa, et al., 2013).

Región Pacífica: esta incluye la zona de vida del bosque muy húmedo tropical (bmh-T), con temperaturas entre los 24 y $28^{\circ} \mathrm{C}$ y altitudes entre 39 y 1.520 metros. Los trabajos se centraron en la diversidad de hormigas en cuatro localidades de la llanura del Pacífico en el municipio de Buenaventura (Chacón de Ulloa, et al., 1996) y la cuenca media del río Calima (Aldana \& Chacón de Ulloa, 1995b; 1999); también se observaron las relaciones existentes en un mosaico de hormigas del bosque lluvioso del Chocó (Armbrecht, et al., 2001a) y se propuso cuantificar la protección que confieren las hormigas mirmicinas (Pheidole) a plantas melastomatáceas del género Tococa (Álvarez, et al., 2001).

Región Amazónica: en esta se evaluaron sistemas productivos de pastizales del departamento del Caquetá con alturas entre los 200 y 400 metros y temperaturas entre los 18 y $36^{\circ} \mathrm{C}$ (Sanabria-Blandón, 2011). Se analizó el gremio de hormigas cazadoras (Sanabria-Blandón \& Chacón de Ulloa, 2011), la riqueza de hormigas legionarias Dorylinae (Ecitoninae) (Sanabria-Blandón \&Achury, 2011) y, además, se reportaron nuevos registros de especies para Colombia (Guerrero \& Sanabria, 2012).

Región de la Orinoquia: la mayoría de los trabajos relativos a esta región se llevaron a cabo en el Centro de Investigación del CIAT en Carimagua, municipio de Puerto Gaitán (Meta), a 200 metros de altitud y una temperatura media de $27^{\circ} \mathrm{C}$, y su objetivo fue registrar las especies de hormigas asociadas a la sabana nativa y a las pasturas introducidas (Medina, et al., 1993; Medina, 1994; Medina, 1995). Igualmente, se evaluó el establecimiento de posibles hormigas indicadoras de los servicios ecosistémicos del suelo en diferentes sistemas productivos del departamento del Meta (Sanabria, et al., 2012).

Región Insular: en esta región se hicieron estudios en las islas de Gorgona y Malpelo, con altitudes entre los 300 y 340 metros y una temperatura promedio de $26{ }^{\circ} \mathrm{C}$. En el Parque Nacional Natural Gorgona se estimaron los cambios ocurridos en un período de 24 años en la fauna de formícidos del suelo y sotobosque, comparando muestreos de fines de la década de los 80 (Baena \&Alberico, 1991) con evaluaciones hechas en el 2010 (Valdés-Rodríguez, et al., 2014); también se registró por primera vez en Colombia información sobre la riqueza y las características particulares de especies de hormigas presentes en el dosel en Gorgona (Chacón de Ulloa, et al., 2014). En el Santuario de Fauna y Flora Malpelo se estudió la composición y la estructura trófica de los invertebrados terrestres (Calero, et al., 2011), entre los cuales se hallaron algunas especies de hormigas introducidas (Calero, et al., 2008).

\section{Resultados y discusión}

Los 60 artículos que ha publicado el Grupo de Investigación en Hormigas en el lapso de 21 años (1993 - 2014) incluyen todas las regiones naturales de Colombia en menor o mayor proporción (Figura 1). La mayor parte de la información se refería a la región Andina, con una representación del $70 \%$ de los estudios, en contraste con el $2 \%$ de la zona Caribe. Los estudios sobre otras regiones naturales (Amazónica, Orinoquia, Pacífica e Insular) tuvieron menor representación, $7 \%$ en cada una, pero incluyeron diferentes ecosistemas.

La ubicación geográfica de la Universidad del Valle ha influido favorablemente en el número de estudios sobre los parches de bosque orientados a evaluar el efecto de la fragmentación sobre la diversidad de las hormigas. La importancia de dicha evaluación radicó en su aporte a los planes de conservación del bosque seco tropical, zona de vida que se encuentra entre los ecosistemas más amenazados del mundo como consecuencia de perturbaciones antropogénicas intensivas (Janzen, 1988; Hoekstra, et al., 2005); en el caso de la cuenca alta del río Cauca, solo queda 1,76 \% de cobertura boscosa (10.716 hectáreas) representada en pequeños fragmentos aislados (Arcila, et al., 2012).

Los trabajos han abarcado diferentes tipos de ambientes (Figura 1S, http://www.raccefyn.co/index.php/raccefyn/ article/downloadSuppFile/124/390); los más estudiados han sido los paisajes rurales, predominantemente los fragmentos de bosque secundario y sus matrices (cultivos, pastizales, guaduales, bosques riparios y corredores). En menor proporción, aunque relevante, han figurado las áreas protegidas del Parque Nacional Natural Gorgona y el Santuario de Fauna y Flora Malpelo (región Insular), el Parque Nacional Natural Farallones de Cali, el Parque Natural Regional El Vínculo y la Reserva Forestal de Bremen (región Andina). En tercer lugar estuvieron los trabajos llevados a cabo en zonas de regeneración y en las principales ciudades del departamento del Valle del Cauca (Cali, Jamundí, Palmira, Buga, Tulúa, Cartago y Buenaventura). Los ecosistemas agrícolas, donde además de la estimación de la diversidad de hormigas, se han registrado especies con potencial para el control biológico (Chacón de Ulloa, 1994), ocuparon el último lugar en cuanto a la representatividad de los hábitats estudiados.

En cada región natural continental (Caribe, Andina, Pacífica, Orinoquia y Amazónica), se estudió un tipo de paisaje y sus respectivos elementos o usos del suelo, con el fin de cuantificar la riqueza y diversidad de la mirmecofauna (Tabla 1). Todos los trabajos evidenciaron una acentuada disminución en la riqueza de las especies de hormigas en elementos del paisaje sin cobertura vegetal natural comparados con los bosques secundarios. Ejemplo de ello es el trabajo de Bustos \& Ulloa (1996, 1997), quienes recolectaron 25 especies de hormigas en tres estados sucesionales de bosque nublado del Parque 
Tabla 1. Riqueza de la mirmecofauna en los diferentes paisajes de las regiones naturales y la zona insular de Colombia. Elemento del paisaje / usos del suelo: bosques (bq), corredor ripario (cr), guadual (gd), potrero (pt), silvopastoriles (sp), cultivos anuales (ca), sustrato rocoso (sr), zona en regeneración (zr), zonas perturbadas (zp), agroforestal (ag), rastrojo (rt), huerto habitacional (hh).

\begin{tabular}{|c|c|c|c|c|}
\hline $\begin{array}{l}\text { Región } \\
\text { natural }\end{array}$ & Tipo de paisaje & Elementos del paisaje - Usos del suelo & $\begin{array}{l}\text { N. de } \\
\text { géneros }\end{array}$ & $\begin{array}{l}\text { N. de } \\
\text { especies }\end{array}$ \\
\hline Caribe & Ecosistemas agrícolas & Cultivos de naranja & 44 & 122 \\
\hline \multirow{5}{*}{ Andina } & Cafetero (Risaralda) & Bosque continuo, parche de bosque y café de sol & 34 & 96 \\
\hline & Cuenca media del río Chambery (Caldas)* & Bosques, corredor ripario, pastizal & 13 & 23 \\
\hline & $\begin{array}{l}\text { Reserva Natural Forestal y de Investigación } \\
\text { Bremen-La Popa (Quindío) }\end{array}$ & Bosque, pastizal & 28 & 58 \\
\hline & Parque Natural Nacional Los Farallones de Cali & Bosque, zona en regeneración & 19 & 25 \\
\hline & Valle interandino del río Cauca & Parches de bosque, guadual, caña de azúcar, potrero & 63 & 215 \\
\hline \multirow{2}{*}{ Pacífica } & Cuenca media del río Calima (Valle del Cauca) & Bosques, zonas en regeneración y perturbadas & 53 & 227 \\
\hline & Cabo Corrientes (Chocó) & Bosque primario & 29 & 117 \\
\hline Amazonía & Piedemonte amazónico (Caquetá) & Agroforestal, huertos, pastos, rastrojo & 42 & 132 \\
\hline \multirow{2}{*}{ Orinoquia } & Sistemas productivos (C.I. Carimagua, Meta) & Pastos mejorados & 19 & 41 \\
\hline & Sistemas productivos del Departamento del Meta & Agroforestal, cultivos anuales, pastizales, sabana & 33 & 92 \\
\hline \multirow{2}{*}{ Insular } & Parque Nacional Natural Gorgona & Bosques, zonas en regeneración & 47 & 130 \\
\hline & Santuario de Fauna y Flora Malpelo & Sustrato rocoso & 3 & 3 \\
\hline
\end{tabular}

* Estudio realizado solo con el grupo de hormigas cazadoras

Nacional Natural Farallones de Cali y encontraron un mayor número de especies en el bosque primario, seguido del bosque secundario y la zona en regeneración.

En el paisaje cafetero de Risaralda se registraron 96 especies de hormigas, 74 de las cuales se hallaron en el bosque continuo, 49 en los parches de bosque y solo 41 en los cafetales a libre exposición (Zabala, et al., 2013). En el valle interandino del río Cauca se ha evaluado el mayor número de elementos del paisaje (fragmentos de bosque secundario, bosques riparios, guaduales, potreros y cultivos, principalmente de caña de azúcar), que, en su conjunto, albergan una importante riqueza regional representada por 215 especies de hormigas, es decir, el $24 \%$ de las conocidas en el país (Chacón de Ulloa, et al., 2012). Debido al muestreo efectuado durante períodos secos y lluviosos a lo largo de varios años, y al empleo de una variedad de métodos complementarios de recolección, el Grupo de Biología, Ecología y Manejo de Hormigas ha podido estudiar profusamente la riqueza de hormigas del bosque seco del valle geográfico del río Cauca. Sin embargo, en los estudios se ha recomendado muestrear el dosel para tener una estimación más acertada de la diversidad de la mirmecofauna (Chacón de Ulloa, et al., 2012).

En los estudios sobre el valle geográfico del río Cauca, los servicios ecosistémicos y el papel ecológico de las hormigas, se evidenciaron las relaciones entre estas y las plantas (Ramírez, et al., 2001) en el control de posibles plagas, principalmente por la depredación que llevan a cabo las hormigas cazadoras (Arcila-Cardona, et al., 2008; Chacón de Ulloa, et al., 2008), y en las interacciones competitivas entre hormigas (Achury, et al., 2008; Achury, et al., 2012).
En la región Pacífica las investigaciones se han centrado en el bosque primario, las zonas en proceso de regeneración y las zonas perturbadas de la cuenca media del río Calima, donde se han registrado un total de 227 especies de hormigas (Tabla 1) que, en su momento, representaron el $33 \%$ de las especies conocidas en Colombia (Aldana \& Chacón de Ulloa, 1999). Además, se ha indagado sobre la importancia de las hormigas dominantes en bosques lluviosos del Chocó biogeográfico tanto desde el punto de vista temporal como espacial (Armbrecht, et al., 2001).

En la región de la Amazónica se evaluaron sistemas productivos del piedemonte llanero y se detectaron nueve tipos de usos del suelo (pasturas tradicionales, sistemas silvopastoriles y agroforestales, cultivos de palma) (Tabla 1). Del grupo de hormigas cazadoras se registraron 35 especies, con una mayor riqueza en la ventana agroforestal (24 especies), mientras que en el sistema silvopastoril se encontraron 19, y en el tradicional, solamente ocho (Sanabria-Blandón \& Chacón de Ulloa, 2012). También se hizo un inventario de hormigas legionarias, grupo de gran importancia para la dinámica de la red trófica en la zona, y se registraron cinco especies en siete tipos de uso del suelo, y se reportaron por primera vez para el departamento de Caquetá las especies Cheliomyrmex andicola y Eciton dulcium (Sanabria \& Achury, 2011).

Se destaca el aporte de los estudios de la zona Insular, donde se ha muestreado el sustrato rocoso del Santuario de Fauna y Flora Malpelo (Calero, et al., 2011). Los estudios reflejan la intensificación del estudio de la mirmecofauna del suelo, sotobosque y dosel en el Parque Nacional Natural Gorgona, incluida la pequeña isla de Gorgonilla (Chacón 
de Ulloa, et al., 2014; Valdés-Rodríguez, et al., 2014). Los investigadores han concluido que después de 24 años de recuperación, Gorgona alberga una gran riqueza (129 especies) (Tabla 1), y que los mecanismos ecológicos y los procesos de recuperación natural han generado microhábitats, permitiendo así la coexistencia de muchas especies (ValdésRodríguez, et al., 2014).

En la Orinoquia se documentó la presencia de 41 especies en sistemas productivos (Tabla 1). Los primeros trabajos reconocieron la fauna asociada a forrajeras tropicales y su implicación como depredadoras de huevos y ninfas del salivazo de los pastos; en los trabajos se destacan las hormigas generalistas de los géneros Solenopsis, Wasmannia y Pheidole (Medina, et al., 1993; Medina, 1994; Medina, 1995). Igualmente, se han evaluado cinco usos del suelo (plantaciones de caucho, de palma, cultivos anuales, sabana nativa y pasturas mejoradas), donde se encontraron 92 especies, 14 de ellas catalogadas como indicadoras de servicios ecosistémicos del suelo, entre las que sobresalían las que anidan en el suelo, dado su efecto sobre la morfología del mismo, y las hormigas vagabundas como indicadoras de la macrofauna (Sanabria, et al., 2012).

Por último, en el Caribe colombiano se reconocieron 122 especies de hormigas en cultivos de naranja con diferentes tipos de manejo ubicados en la zona de vida de bosque seco tropical (Tabla 1S, http://www.raccefyn.co/index.php/raccefyn/ article/downloadSuppFile/124/378). Además de evidenciar la sensibilidad de las hormigas a los agroquímicos, se destacó el grupo de las hormigas cazadoras y las mirmicinas crípticas como potenciales controladores biológicos de las termitas que afectan los árboles de cítricos (Abadía, et al., 2013).

\section{Especies raras, comunes, endémicas e introducidas}

En los estudios sobre las diferentes regiones se mencionan con especial énfasis las especies de hormigas que aportan información sobre el estado de equilibrio y heterogeneidad de la localidad evaluada (Tabla 1S). Se señaló a la subfamilia Myrmicinae como la más diversa y la que contiene el mayor número de especies consideradas raras, endémicas, comunes o introducidas; le siguieron las subfamilias Ponerinae, Formicinae y Dolichoderinae, en tanto que de las subfamilias más pequeñas, Amblyoponinae, Cerapachynae y Proceratiinae, solamente se hallaron una o dos especies. De las 34 especies raras (aquellas presentes solo en un hábitat de un elemento del paisaje), el 26,5\% correspondió a mirmicinas, especialmente de la especie en peligro de extinción Oxyepoecus inquilinus (Fernández, 2002), la cual se halló en la región Caribe (Abadía, et al., 2013). En segundo lugar se registró la subfamilia Ponerinae $(20,6 \%)$, con cinco especies de hormigas cazadoras del género Pachycondyla. Con un menor porcentaje $(11,8 \%)$ se menciona el género Gracilidris (Dolichoderinae), con su única especie Gracilidris pombero, en la región de la Amazonia (Guerrero \& Sanabria, 2012). Es importante resaltar que, según los estudios, la mayoría de las especies consideradas raras se recolectaron en hábitats con cobertura boscosa (Bustos \& Ulloa-Chacón, 1996-97; Armbrecht \& Chacón de Ulloa, 1997; Armbrecht \& Chacón de Ulloa, 1999; Aldana \& Chacón de Ulloa, 1999; Arcila-Cardona, et al., 2008; Chacón de Ulloa, et al., 2008; Chaves, et al., 2008; Zabala, et al., 2008; Abadía, et al., 2010; Chacón de Ulloa, et al., 2012; Zabala, et al., 2013; Chacón de Ulloa, et al., 2014; Valdés-Rodríguez, et al., 2014).

En el ecosistema de bosque seco del río Cauca, los bosques en su conjunto aportaron más especies propias y raras que los otros hábitats (bosques riparios, guaduales, cultivos, potreros), por lo que se ha propuesto que la conservación de uno o pocos fragmentos boscosos no es suficiente para la preservación de la fauna de hormigas en el valle del río Cauca y que, por el contrario, conservar todos los fragmentos es un punto clave para el mantenimiento de la diversidad regional (Armbrecht \& Ulloa-Chacón, 1999; Chacón de Ulloa, et al., 2012).

Entre las 30 especies consideradas comunes (Tabla 1S), es decir, aquellas presentes en casi todos los hábitats y de amplia distribución geográfica, en los estudios se mencionan las mirmicinas generalistas, como la hormiga nativa de fuego Solenopsis geminata, las hormigas cabezonas Pheidole biconstricta, Pheidole radoszkowskii, Pheidole susannae, y la pequeña hormiga de fuego Wasmannia auropunctata (Armbrecht \& Chacón de Ulloa, 1997; Armbrecht \& Chacón de Ulloa, 1999; Armbrecht \& Ulloa-Chacón, 2003; Achury, et al., 2011; 2012; Chacón de Ulloa, et al., 2012; Abadía, et al., 2013; Zabala, et al., 2013). Este grupo de hormigas se encuentra frecuentemente en ecosistemas perturbados, en zonas muy intervenidas, como jardines y terrenos baldíos, en áreas urbanas y campos cultivados, donde encuentran un entorno apropiado para incrementar sus poblaciones y dominar aprovechando eficientemente los recursos (Chacón de Ulloa, 2003; Chaves, et al., 2008; Jiménez, et al., 2008; Achury, et al., 2012; Chacón de Ulloa, et al., 2012).

Entre las hormigas cazadoras más comunes objeto de los estudios sobresale la hormiga cachona Ectatomma ruidum en los Llanos Orientales (Medina, 1995), en varias localidades del bosque seco tropical del valle geográfico del río Cauca y en cultivos de naranja de la región Caribe (Arcila-Cardona, et al., 2008; Chacón de Ulloa, et al., 2012; Abadía, et al., 2013). Otros autores han descrito esta especie como posible agente de control natural en pasturas de la región Andina (Santamaría, et al., 2009).

El 35,6 \% de las especies endémicas son ectatominas del género Gnamptogenys (Tabla 1S) y se encuentran principalmente en los bosques lluviosos del Chocó biogeográfico 
(Fernández \& Sendoya, 2004; Zabala, et al., 2008). El Pacífico colombiano es una de las regiones con mayor riqueza en recursos naturales y con altos índices de biodiversidad y endemismos, ya que posee una de las selvas primarias tropicales más exuberantes y diversas del mundo (Zabala, $\boldsymbol{e t}$ al., 2008). Se ha explicado que eventos como el levantamiento de los Andes, particularmente el de la cordillera Occidental, tuvieron gran efecto sobre la distribución y diversificación de la biota, aislándola progresivamente y sometiéndola a diferentes condiciones climáticas por el efecto de sombra de lluvia (Zabala, et al., 2008). Igualmente, los refugios pleistocénicos ubicados en el Chocó biogeográfico han sido centros de especiación y endemismo importantes y han permitido la diversificación de grupos como Gnamptogenys y Pachycondyla (Zabala, et al., 2008; Baena, 1993).

Por último, entre las especies introducidas se destacan las hormigas oportunistas con hábitos generalistas que pueden llegar a comportarse como invasoras; es el caso de la hormiga loca Nylanderia fulva, que fue introducida a Colombia en la década de los años 70 y llegó a distribuirse en ocho departamentos (Chacón de Ulloa, et al., 1994; Aldana, et al., 1995a; Arcila \& Quintero, 2005; Chacón de Ulloa, et al., 2006).

\section{Especies indicadoras}

Hormigas identificadas hasta el nivel específico se han propuesto como especies indicadoras de los elementos del paisaje estudiados en cada región natural (Tabla $2 \mathrm{~S}$, http://www.raccefyn.co/index.php/raccefyn/article/ downloadSuppFile/124/392). En la mayoría de los trabajos la atención se enfocó en el grupo de las hormigas cazadoras, pues debido a sus requerimientos de microhábitat (nidifican entre hojarasca y madera en descomposición), son susceptibles a los cambios en el ambiente (Arcila-Cardona, et al., 2008; Chacón de Ulloa, et al., 2008; Chaves, et al., 2008; Abadía, et al., 2010; Sanabria-Blandón \& Chacón de Ulloa, 2011; Cabra-García, et al., 2012). En los trabajos se han propuesto cuatro especies para el bosque primario de la región Andina, entre las que se destacan dos del género Heteroponera (H. inca y H. monticola) (Bustos \& Ulloa-
Chacón, 1996-97; Armbrecht \& Chacón de Ulloa, 1997; Armbrecht \& Chacón de Ulloa, 1999;Abadía, et al., 2010). Por el contrario, para el corredor ripario solo se menciona la especie Gnamptogenys bisulca; para el guadual, Mayaponera (Pachycondyla) constricta, y para las zonas en regeneración, Labidus preadator (Bustos \& Ulloa-Chacón, 1996-97; Arcila-Cardona, et al., 2008; Abadía, et al., 2010).

En la región natural del Pacífico también se han seleccionado especies bioindicadoras, principalmente de bosques poco perturbados, como Acanthoponera mucronata, Belonopelta deletrix, Cyphomyrmex cornutus, Dolichoderus shattucki y Odontomachus cornutus (Aldana \& Chacón de Ulloa, 1999). En la Amazonia, diez especies de hormigas resultaron indicadoras de hábitats con cobertura arbórea, sobresaliendo tres del género Pachycondyla y la hormiga conga Paraponera clavata, en tanto que en el sistema silvopastoril se mencionó la hormiga carpintera Camponotus simillimus indianus (Sanabria, 2011; Sanabria-Brandón \& Chacón de Ullloa, 2011).

En la Orinoquia se han registrado Hypoponera punctatissima y Pseudomyrmex gracilis como especies con valor indicador para el uso del suelo de cultivos anuales (arroz, maíz y soya). En zonas muy perturbadas se destacan aquellas hormigas vagabundas y oportunistas de los géneros Pheidole, Solenopsis y Wasmannia (Armbrecht \& Chacón de Ulloa, 1997; Aldana \& Chacón de Ulloa, 1999; Armbrecht \& Chacón de Ulloa, 1999; Armbrecht \& Ulloa-Chacón, 2003; Achury, at el., 2011). Además, la pequeña hormiga de fuego $W$. auropunctata se ha propuesta como indicadora negativa de la riqueza y abundancia de hormigas del bosque seco (Armbrecht \& Ulloa-Chacón, 2003; Achury, et al., 2012).

En conclusión, mediante el estudio de la ecología, el comportamiento, los servicios ecosistémicos, la distribución y la historia natural de las hormigas, el Grupo de Investigación en Biología, Ecología y Manejo de Hormigas de la Universidad del Valle ha contribuido al conocimiento de su diversidad en el país y a demostrar su valor como herramienta eficiente y rápida para la conservación e identificación del estado de un hábitat o paisaje.

\section{Información suplementaria}

Figura 1S. Tipos de ambientes explorados en estudios de la mirmecofauna de Colombia

Tabla 1S. Especies de hormigas que revisten mayor importancia en los paisajes estudiados en las regiones naturales de Colombia: Caribe (ca), Andina (an), Pacífica (pa), Orinoquia (or), Amazónica (am), Insular (in)
Tabla 2S. Especies de hormigas con valor significativo como indicadoras de los elementos del paisaje en las diferentes regiones naturales de Colombia 


\section{Agradecimientos}

A todas las personas que durante 20 años han contribuido al desarrollo de los diferentes proyectos y a la elaboración de los trabajos que se recopilan en el presente escrito. Al Departamento de Biología de la Universidad del Valle, por su apoyo económico y de logística; a las siguientes instituciones por la cofinanciación de las investigaciones: Colciencias, Ministerio de Agricultura y Desarrollo Rural, Banco Mundial, Instituto Humboldt, National Geographic, National Science and Engineering Research Council Canada, Invemar, Corpoica, Wild Life Conservation Society, Fundación Farallones de Cali, Fundación José Celestino Mutis (FEN de Colombia). A David Calero, por la elaboración del mapa, a Carmen Elisa Posso, por sus valiosos aportes al manuscrito, y a Philip Silverstone-Psokin, por la revisión del resumen en inglés.

\section{Bibliografía}

Abadía, J. C., Bermúdez, C., Lozano-Zambrano, F. \& Chacón, P. 2010. Hormigas cazadoras en un paisaje subandino de Colombia: riqueza, composición y especies indicadoras. Revista Colombiana de Entomología 36 (1): 127-134.

Abadía, J. C., Arcila, A. M. \& Chacón de Ulloa, P. 2013. Hormigas en cultivos de naranja (Citrus sinensis (L.) Osbeck) de la costa Caribe de Colombia. Biota Colombiana 14 Suplemento especial - Artículos de datos: 13-19.

Achury, R., Chacón de Ulloa, P. \& Arcila, A. M. 2008. Composición de hormigas e interacciones competitivas con Wasmannia auropunctata en fragmentos de bosque seco tropical. Revista Colombiana de Entomología 34 (2): 209-216.

Achury, R., Arcila, A. M. \& Chacón de Ulloa, P. 2011. The Myrmicinae ant Pheidole susannae as potencial urban pest in Colombia. En: Proceeding of the $7^{\text {th }}$ International Conference on Urban Pests. (Robinson, W. H. \& De Carvalho-Campos, A. E. (eds.). OuroPreto, Brazil. 417 p.

Achury, R., Chacón de Ulloa, P. \& Arcila, A. 2012. Effects of the Heterogeneity of the Landscape and the Abundance of Wasmannia auropunctata on Ground Ant Assemblages in a Colombia Tropical Dry Forest. Psyche. Doi:10.1155: 1.12.

Aldana, R. C., Baena, M. \& Chacón de Ulloa, P. 1995a. Introducción de la hormiga loca (Paratrechina fulva) a la Reserva Natural Laguna de Sonso (Valle del Cauca, Colombia). Bol Mus Ent Universidad Valle 3 (1): 15-28.

Aldana, R. C. \& Chacón de Ulloa, P. 1995b. Nuevos registros de hormigas (Hymenoptera: Formicidae) para Colombia. Bol Mus Ent Universidad Valle 3 (2): 55-59.

Aldana, R. C. \& Chacón de Ulloa, P. 1999. Megadiversidad de hormigas (Hymenoptera: Formicidae) de la cuenca media del río Calima. Revista Colombiana de Entomología 25 (1-2): $37-47$.

Álvarez, G., Armbrecht, I., Jiménez, E., Armbrecht, H. \& UlloaChacón, P. 2001. Ant-plant association in two Tococa species from a primary rain forest of Colombian Chocó (Hymenoptera: Formicidae). Sociobiology 38: 585-602.

Alonso, L. E. 2000. Ants as indicators of diversity. En: Ants standard methods for measuring and monitoring biodiversity. Agosti, D., Majer, J., Alonso, L. \&Schultz, T. (eds.). Smithsonian Institution Press, Washington. 304 p.

Andersen, A. N. \& Majer, J. D. 2004. Ants show the way DownUnder: Invertebrates as bioindicators in land management. Frontiers in Ecology and the Environment 2: 291-298.

Arcila, A., Gómez, L. A. \& Ulloa-Chacón, P. 2002a. Immature development and colony growth of crazy ant Paratrechina fulva (Mayr) under laboratory conditions. Sociobiology 39 (2): 307-321.

Arcila, A., Ulloa-Chacón, P. \& Gómez, L. A. 2002b. Factors that influence fecundity of queens and queen production in crazy ant Paratrechina fulva (Hymenoptera: Formicidae). Sociobiology 39 (2): 323-334.

Arcila, A \& Quintero, M. 2005. Impacto e historia de la introducción de la hormiga loca (Paratrchina fulva) a Colombia. Informe final, contrato de prestación de servicios No. 136 Instituto de Investigación de Recursos Biológicos Alexander von Humboldt $91 \mathrm{p}$.

Arcila-Cardona, A., Osorio, A. M., Bermúdez, C. \& Chacón de Ulloa, P. 2008. Diversidad de hormigas cazadoras asociadas a los elementos del paisaje del bosque seco. En: Sistemática, biogeografía y conservación de las hormigas cazadoras de Colombia. Lozano-Zambrano, F., Fernández, F., Jiménez, E. \& Árias, T. (eds.). Instituto de Investigaciones de Recursos Biológicos Alexander von Humboldt. Bogotá D. C. Colombia. 617 p.

Arcila Cardona, A. M., Valderrama Ardila, C. \& Chacón de Ulloa, P. 2012. Estado de fragmentación del bosque seco de la cuenca alta del río Cauca. Biota Colombiana, especial bosque seco en Colombia 13 (2): 86-100.

Arenas, A., Armbrecht, I. \& Chacón, P. 2013. Carábidos y hormigas del suelo en dos áreas cultivadas con maracuyá amarillo (Passiflora edulis) en el Valle del Cauca, Colombia. Acta Biológica Colombiana 18 (3): 439-448.

Armbrecht, I. \& Chacón de Ulloa, P. 1997. Composición y diversidad de hormigas en bosques secos relictuales y sus alrededores, en el Valle del Cauca, Colombia. Revista Colombiana de Entomología 23 (1-2): 45-50.

Armbrecht, I. \& Ulloa-Chacón, P. 1999. Rareza y diversidad de hormigas en fragmentos de bosque seco colombianos y sus matrices. Biotropica 31 (4): 646-653.

Armbrecht, I., Jiménez, E., Álvarez, E. G., Ulloa-Chacón, P. \& Armbrecht, H. 2001a. An ant mosaic in the Colombian rain forest of Chocó (Hymenoptera: Formicidae). Sociobiology 37 (3B): 491-509.

Armbrecht, I., Tischer, I. \& Chacón, P. 2001b. Nested subsets and partition patterns in ant assemblages (Hymenoptera, Formicidae) of Colombian dry forest fragments. Pan Pacific Entomologist 77 (3): 196-209. 
Armbrecht, I. \& Ulloa-Chacón, P. 2003. The little fire ant Wasmannia auropunctata (Roger) (Hymenoptera: Formicidae) as a diversity indicator of ants in tropical dry forest fragments of Colombia. Environmental Entomology 32 (3): 542-547.

Baena, M. L. 1993. Hormigas cazadoras del género Pachycondyla (Hymenoptera: Ponerinae) de la Isla Gorgona y la planicie pacífica colombiana. Bol Mus Ent. Universidad Valle 1: 13-21.

Baena, M.L. \& M. Alberico. 1991. Relaciones biogeográficas de las hormigas de la Isla Gorgona. Revista Colombiana de Entomología 17: 24-31.

Brown Jr. K. S. 1991. Conservation of neotropical environment: Insects as indicators. In: Conservation of insects and their habitats. Collins, N. M. \& Thomas, J. (eds.). London, Academic Press. 430 p.

Bustos, J. \& Ulloa-Chacón, P. 1996-97. Mirmecofauna y perturbación en un bosque de niebla neotropical (Reserva Natural Hato Viejo, Valle del Cauca, Colombia). Revista de Biología Tropical 44 (3)/45 (1): 259-266.

Bolton, B. 2007. Taxonomy of the dolichoderine ant genus Technomyrmex Mayr (Hymenoptera: Formicidae) based on the worker caste. Contributions of the American Entomological Institute 35 (1):1-150.

Brady, S.G., Schultz, T. R., Fisher, B.L. \& Ward, P. S. 2006. Evaluating alternative hypotheses for the early evolution and diversification of ants. Proceedings of the National Academy of Sciences of the United States of America 103: 18172-18177.

Cabra-García, J. C., Bermúdez, C., Osorio, A. M., \& Chacón, P. 2012. Cross-taxon congruence of $\gamma$ and $\beta$ diversity among five leaf litter arthropod groups in Colombia. Biodiversity and Conservation. Doi 10.1007/s10531-012-0259-5.

Calero, D., López-Victoria, M. \& Chacón, P. 2008. Alien species on a very isolated island: The case of Malpelo, 35 years after the first invertebrate records. In: Towards a synthesis: Neobiota book of abstracts. Pyseck, P. \& Pergl, J. (eds.). Institute of Botany Pruhonice, Academy of Sciences, Czech Republic. 250 p.

Calero, D., López-Victoria M. \& Chacón de Ulloa, P. 2011. Composición y estructura trófica de los invertebrados terrestres de la Isla Malpelo, Pacífico colombiano. Boletín de Investigaciones Marinas y Costeras 40 (supl. Esp.): 155-173.

Chacón de Ulloa, P. 1994. Biología e impacto económico de las hormigas. Palmas 15 (4): 25-30.

Chacón de Ulloa, P. 1998. Introducción de la hormiga loca en Colombia. En: Informe Nacional sobre el estado de la biodiversidad. Causas de Pérdida de la Biodiversidad. Chaves, M.E. \& Arango, N. Instituto de Investigación de Recursos Biológicos Alexander von Humboldt. Bogotá, 99-100 p.

Chacón de Ulloa, P. 2003a. Biología Reproductiva de Wasmannia auropunctata (R.) (Hymenoptera: Formicidae). Revista de la Academia Colombiana de Ciencias Exactas, Físicas y Naturales 27 (104): 441-447.

Chacón de Ulloa, P. 2003b. Hormigas urbanas. En: Introducción a las hormigas de la región Neotropical. Fernández, F. (ed.). Instituto de Investigación de Recursos Biológicos Alexander von Humboldt. Bogotá. 424 p.

Chacón de Ulloa, P., Baena, M. L. \&Aldana, R. C.1994. Efecto de dos análogos de la hormona juvenil, fenoxycarb y metopreno, sobre la hormiga loca Paratrechina fulva (Hymenoptera: Formicidae). Revista Colombiana de Entomología 20 (3): 193-198.

Chacón de Ulloa, P., Baena, M. L., Bustos, J., Aldana, R. C. \& Gamboa, M. 1996. Fauna de hormigas del departamento del Valle del Cauca. En: Insectos de Colombia. Estudios escogidos. Andrade, G. Amat-García, G. Fernández, F. (eds.). Academia Colombiana de Ciencias Exactas, Físicas y Naturales. Colección Jorge Álvarez Llenar Número 10. Coedición con el Centro Editorial Javeriano. Bogotá, Colombia. 413-451 p.

Chacón de Ulloa, P., Bustos, J., Aldana, R. C. \& Baena, M. L. 2000. Control de la hormiga loca, Paratrechina fulva (Hymenoptera: Formicidae) con cebos tóxicos en la Reserva Natural Laguna de Sonso (Valle, Colombia). Revista Colombiana de Entomología 26 (3-4): 151-156.

Chacón de Ulloa, P. \& Armbrecht. I. 2006. Las hormigas del Bosque seco Tropical. En: Informe sobre el avance en el conocimiento y la información de la biodiversidad 19982004. Chávez, M. E. \& Santamaría, M. (eds.). Instituto de Investigación de Recursos Biológicos Alexander von Humboldt. Bogotá. 459 p.

Chacón de Ulloa, P., Jaramillo, G. I. \& Lozano, M. M. 2006. Hormigas urbanas en el departamento del Valle del Cauca, Colombia. Revista de la Academia Colombiana de Ciencias Exactas, Físicas y Naturales 30 (116): 435- 441.

Chacón de Ulloa, P., Armbrecht, I. \& Lozano-Zambrano. 2008. Aspectos de la ecología de hormigas cazadoras en bosques secos colombianos. En: Sistemática, biogeografia y conservación de las hormigas cazadoras de Colombia. Lozano-Zambrano, F., Fernández, F., Jiménez, E. \& Arias, T. (eds.). Instituto de Investigaciones de Recursos Biológicos Alexander von Humboldt. Bogotá D. C. Colombia. 617 p.

Chacón de Ulloa, P. \& Achury, R. 2011. The introduced big-headed ant Pheidole megacephala Forel in southern Colombia. En: Proceeding of the $7^{\text {th }}$ International Conference on Urban Pests. (Robinson, W. H. \& De Carvalho-Campos, A. E. (eds.). Ouro Preto, Brazil. 417 p.

Chacón de Ulloa, P., Osorio, A. M., Achury, R. \& Bermúdez, C. 2012. Hormigas (Hymenoptera: Formicidae) del bosque seco tropical de la cuenca alta del río Cauca (Colombia). Biota Colombiana 13 (2): 165-181.

Chacón de Ulloa, P., Ramírez, L. \& Rodríguez, M. 2013. Capítulo Colombia. En: Ecología Urbana: Experiencias en América Latina. Ortega, R. \& McGregor-Fors, I. (eds.). Instituto de Ecología, México. 130 p. 
Chacón de Ulloa, P., Valdés-Rodríguez, S., Hurtado-Giraldo, A \& Pimienta, M. C. 2014. Hormigas arbóreas del Parque Nacional Natural Gorgona (Colombia). Revista de Biología Tropical 62 (Sup. 1): 227-287.

Chaves, M. C. 2003. Riqueza y composición de especies de la comunidad de hormigas del suelo (Hymenoptera: Formicidae) en un gradiente espacial bosque-borde-matriz de pastizal. Tesis de pregrado. Universidad del Valle, Cali. Colombia. $65 \mathrm{p}$.

Chaves, M. C., Chacón de Ulloa, P. \& Lozano-Zambrano, F. 2008. Riqueza y rareza de hormigas cazadoras en el gradiente bosque-borde-pastizal de un fragmento de bosque subandino (Quindío, Colombia). En: Sistemática, biogeografía y conservación de las hormigas cazadoras de Colombia. Lozano-Zambrano, F., Fernández, F., Jiménez, E. \& Arias, T. (eds.). Instituto de Investigaciones de Recursos Biológicos Alexander von Humboldt. Bogotá D. C. Colombia. 617 p.

Fernández, F. 2002. New ant records for Colombia and South America Hymenoptera Formicidae. Revista Colombiana de Entomología 28 (2): 215.

Fernández, F. (Ed.). 2003. Introducción a las hormigas de la región neotropical. Instituto de Investigación de Recursos Biológicos Alexander von Humboldt, Bogotá D. C. Colombia. 398 p.

Fernández, F. \& Sendoya, S. 2004. Lista de las hormigas neotropicales. Biota Colombiana 5 (1): 3-109.

García, R., Armbrecht, I., Ulloa-Chacón, P. 2001. Staphylinidae (Coleoptera): composición y mirmecofilia en bosques secos relictuales de Colombia. Folia Entomológica Mexicana 40 (1): 1-10.

García, R. \& Chacón de Ulloa, P. 2005. Estafilínidos (Coleoptera: Staphylinidae) en fragmentos de bosque seco del valle geográfico del río Cauca. Revista Colombiana de Entomología 31 (1): 43-50.

Guerrero, R. J. \& Sanabria, C. 2012. The first record of the genus Gracilidris (Hymenoptera: Formicidae: Dolichoderinae) from Colombia. Revista Colombiana de Entomología 37 (1): 159-161.

Hoekstra, J., Boucher, T., Ricketts, T. \& Roberts. C. 2005. Confronting a biome crisis: Global disparities of habitat loss and protection. Ecology Letters 8: 23-29.

Hölldobler, B. \& Wilson, E. O. 1990. The ants. Harvard University Press, Cambridge, Massachusetts. 732 p.

Janzen, D. 1988. Tropical dry forests. The most endangered major tropical ecosystem. In: Biodiversity. Wilson, E. O. (ed.). National Academy of Sciences, Smithsonian Institution. Washington. $538 \mathrm{p}$.

Jaramillo, G. I. \& Chacón de Ulloa, P. 2003. La hormiga fantasma Tapinoma melanocephalum (Hymenoptera: Formicidae): fecundidad de reinas y desarrollo de colonias experimentales. Revista Colombiana de Entomología 29 (2): 227-230.

Jones, T. H., Wosciechowski, T. J., Snelling, R., Torres, J. A., Chacón, P. \& Devries, P. J. 1999. Dialkylpyrrolidines from the ants Megalomyrmex cyendyra $\mathrm{B}$. and $M$. latreille E. Caribbean Journal of Science 35 (3-4): 310-311.

López, M. P., Arcila, A. M. \& Chacón de Ulloa, P. 2008.Ausencia del parasitoide Orasema minutissima en poblaciones de la hormiga Wasmannia auropunctata del sur occidente de Colombia. Bol Mus Ent Universidad Valle 9 (1): 17-21.

Lozano-Zambrano, F. H., Ulloa-Chacón, P. Armbrecht, I. 2009. Hormigas: relaciones especies-área en fragmentos de bosque seco tropical. Neotropical Entomology 38 (1): 44-54.

Majer, J. D. 1983. Ants: Bio-indicators of minesite rehabilitation, land use and land conservation. Environmental Management 7: $375-383$

Medina, C. A., Chacón de Ulloa, P. \& Lapointe, S. 1993. Fauna de hormigas en pasturas introducidas y sabana nativa, Carimagua, LLanos Orientales de Colombia. Revista Colombiana de Entomología 19 (4): 143-150.

Medina, C. A. 1994. Nidificación y patrones de distribución espacial de nidos de hormigas en una sabana tropical, Carimagua: Llanos Orientales de Colombia. Bol Mus Ent. Universidad Valle $2(1,2): 31-42$.

Medina, C. A. 1995. Hormigas depredadoras de huevos de salivazo de los pastos Aeneolamia varia (Hemiptera: Cercopidae) en pasturas de Brachiaria, en los Llanos Orientales de Colombia. Bol Mus Ent Universidad Valle (1): 1-13.

Montoya, J. P., Chacón de Ulloa P. \& Manzano M. R. 2006. Caracterización de nidos de la hormiga arriera Atta cephalotes (Hymenoptera: Myrmicinae) en Cali (Colombia). Revista Colombiana de Entomología 32 (2): 151-158.

Olaya, L. A., Chacón de Ulloa, P. \& Payán, A. 2005. Hormigas (Hymenoptera: Formicidae) en centros hospitales del Valle del Cauca como vectores de patógenos nosocomiales. Revista Colombiana de Entomología 31 (2): 183-187.

Ramírez, M., Chacón de Ulloa, P., Armbrecht, I. \& Calle, Z. 2001. Contribución al Conocimiento entre plantas, hormigas y homópteros en bosques secos de Colombia. Caldasia 23 (2): 523-536.

Sanabria-Blandón, M. C. \& Chacón de Ulloa, P. 2009. Hormigas como plagas potenciales en tres criaderos de mariposas del suroccidente de Colombia. Acta Agronómica 58 (1): 47-52.

Sanabria-Blandón , M. C. 2011. Ensamblaje de hormigas del suelo en ventanas productivas del piedemonte amazónico colombiano. Tesis de maestría, Universidad del Valle. Cali, Colombia. 89 p.

Sanabria-Blandón, M. \& Achury, R. 2011. Hormigas legionarias (Formicidae: Ecitoninae) en sistemas productivos de Caquetá (Colombia). Acta biológica Colombiana 16 (1): 219-224.

Sanabria-Blandón, M.C. \& Chacón de Ulloa, P. 2011. Hormigas cazadoras en sistemas productivos del piedemonte amazónico colombiano: diversidad y especies indicadoras. Acta Amazonica 41 (4): 503-512. 
Sanabria, C., Chacón, P., Rodríguez, E. A. \& Lavelle, P. 2012. Soil ants as indicators of provision of ecosystem services in production system of the Eastern plains of Colombia. En: XVI Congreso colombiano de la ciencia del suelo "La Ciencia del Suelo al Servicio de la Sociedad y del Ambiente". Sociedad Colombiana de la Ciencia del suelo. Riohacha, Colombia. 611 p.

Santamaría, C., Armbrecht, I. \& Lachaud, J. P. 2009. Nest distribution and food preferences of Ectatomma ruidum (Hymenoptera: Formicidae) in shaded and open cattle pastures of Colombia. Sociobiology 53 (2): 1-25.

Ulloa-Chacón, P. \& Jaramillo, G. 2003. Effects of boric acid, fipronil, hydramethylnon and diflubenzuron on colonies of the ghost ants (Hymenoptera: Formicidae). Journal of Economic Entomology 96 (3): 856-862.

Valdés-Rodríguez, S., Chacón de Ulloa, P. \& Armbrecht, I. 2014. Riqueza de hormigas del suelo en el Parque Nacional
Natural Gorgona, Pacífico colombiano. Revista de Biología Tropical 62 (Sup 1): 265-276.

Ward, P. S. 2007. Phylogeny, classification, and species-level taxonomy of ants (Hymenoptera: Formicidae). Zootaxa 1668: $549-563$.

Zabala, G. A., Gutiérrez, C. \& Chacón de Ulloa, P. 2008. Biogeografía provincial: ponerofauna del Valle del Cauca. En: Sistemática, biogeografía y conservación de las hormigas cazadoras de Colombia. Lozano-Zambrano, F., Fernández, F., Jiménez, E. \& Arias, T. (eds.). Instituto de Investigaciones de Recursos Biológicos Alexander von Humboldt. Bogotá D. C. Colombia. 617 p.

Zabala, G. A., Arango, L. M. \& Chacón de Ulloa. 2013. Diversidad de hormigas (Hymenoptera: Formicidae) en un paisaje cafetero de Risaralda, Colombia. Revista Colombiana de Entomología 39 (1): 141-149. 